\title{
Strategies for Combined Operation of PV/Storage Systems Integrated into Electricity Markets
}

\author{
Thomas Carriere ${ }^{1,3}$, Christophe Vernay ${ }^{2}$, Sebastien Pitaval ${ }^{2,3}$, Francois-Pascal Neirac ${ }^{1}$, George \\ Kariniotakis ${ }^{1}$ \\ ${ }^{1}$ MINES ParisTech, PSL University, Centre PERSEE, CS 10207, rue Claude Daunesse, 06904 Sophia Antipolis Cedex, France \\ 2 SOLAÏS, 55 allée Pierre Ziller, 06560 Sophia Antipolis Cedex, France \\ ${ }^{3}$ Third Step Energy, 55 allée Pierre Ziller, 06560 Sophia Antipolis Cedex, France \\ *E-mail: thomas.carriere@mines-paristech.fr
}

\begin{abstract}
The increasing share of photovoltaic (PV) power in the global energy mix presents a great challenge to power grid operators. In particular, PV power's intermittency caused by varying weather conditions can lead to mismatches between energy production and expectation. Battery Energy Storage Systems (BESS) are often put forward as a good technological solution to these problems, as they are able to mitigate PV power forecast errors. However, the investment cost of such systems is still high, which questions the benefits in relation to the cost of using these systems in operational contexts. In this paper, we compare several strategies to manage a PV power plant coupled with a BESS in a market environment. They are obtained by stochastic optimization using a Model Predictive Control (MPC) approach. This paper proposes an approach that takes into account the aging of the BESS, both at the day-ahead level and in the real-time control of the BESS, by modeling the cost associated with BESS usage. As a result, the BESS arbitrates between compensating forecast errors and preserving its own life expectancy, based on both PV production and price scenarios derived from probabilistic forecasts. A sensitivity analysis is also carried out to provide guidelines on the optimal sizing of the BESS capacity, depending on market characteristics and BESS prospective costs.
\end{abstract}

\section{Introduction}

The significant share of PV power employed in several countries poses challenges due to the discrepancies between expected and actual energy production. This is a major issue for Transmission System Operators (TSO), which have to ensure that energy production and demand always match. Most countries apply policies whereby the risk is borne by the PV plant operators, which pay the TSO for any discrepancies between their day-ahead forecast production and actual production.

Thus, the derivation of optimal bids on a day-ahead electricity market for intermittent energy production resources has been an active field of research in recent years. In [1] and [2], the authors proposed different ways of deriving bids using probabilistic forecasts of the production. Reference [3] developed these ideas and proposed analytical solutions to the optimal bidding problem. However, the revenue of a given producer is still quite sensitive to the uncertainty of the power generation resource, and the financial penalties caused by forecast errors can represent a significant loss for the producer.

BESS units are viewed as a good technical solution to deal with these problems, thanks to their ability to compensate for forecast errors. However, these systems are still costly and their actual financial benefit is difficult to quantify in the long term, which makes PV plant operators reluctant to install them. Thus, it is very important to define strategies that ensure the optimal operation of a PV/BESS system, so that the benefit of installing a storage system is maximized. Two strategies are required: one for the day-ahead level when bids are submitted to the electricity market, and one for the real-time control of the BESS.

Several papers propose bidding strategies at the day-ahead level for standalone large-scale BESS [4], [5]. Although they report good performance, the optimal offering strategy is largely dependent on market design. Besides, these authors assume that a BESS can freely

Paper submitted for publication at IET Renewable Power Generation journal charge or discharge on the grid, which is not the case when considering combined PV/BESS. An interesting result from [5] is that maximizing the BESS owner's profit results in reduced social welfare, which shows that the price signals do not necessarily work in favor of the grid's operation. In [6], the authors propose a bidding method for a combined wind/hydro plant, but they assume perfect wind forecasts at the operation stage. Reference [7] also proposes a method for a wind/hydro plant for both the day-ahead and operational stages, but they do not attempt to model the market prices because of their high volatility.

The standard method for real-time control of PV/BESS is Model Predictive Control (MPC). This consists in optimizing the control of the BESS on a receding horizon, in order to take into account the forecast future state of the system when optimizing the next time step. Different loss functions can be optimized on the receding horizon. In most cases, the optimized function is either the producer's profit [8], [9] or the energy imbalance [10], that is the deviation between the planned schedule and the actual outcome, without taking profit into consideration. Some authors also propose an MPC approach to bid on intra-day market sessions [11], [12]. The uncertainty of the upcoming PV production is sometimes included in both the day-ahead planning and the real-time control of the BESS, as in [13], [14] or [15].

Overall, several points are often neglected in the literature. The first is the aging cost of a BESS. This is mentioned in [9], where constraints are defined to improve the life expectancy of the BESS, and in [12] where the MPC loss function is penalized by the total amount of energy flowing in and out of the BESS. At the day-ahead level, [4] considers aging with a finer modeling. While few papers consider the uncertainty of renewable energy forecasts, [5] and [16] model this uncertainty using production scenarios.

We include all of these elements to propose and compare different strategies for controlling a PV/BESS system. These strategies include all of the elements that lacked in the previous approaches. Deterministic forecasts of both day-ahead and balancing prices are performed, along with probabilistic forecasts of the PV power production. In particular, we establish that it is very important to use a representation of the uncertainty based on production scenarios, 
derived from the forecasts. Using a sequence of forecast distributions fails to account for the temporal correlation of the forecast errors, which is critical to the good operation of a storage system. In addition, a model of the cost associated with the BESS aging is implemented to take it into account at both day-ahead and real-time decision levels. Most of the strategies are obtained by stochastic optimization using an MPC approach, but we also provide analytical solutions after simplifying the bidding problem.

The key contributions of the paper are (1) the inclusion of the BESS usage cost as a penalization of the revenue when proposing bids or managing the BESS in real time; (2) the derivation of a closed-form analytic solution to the market penalty minimization problem at the real-time level. This allows us to extend analytic solutions in the literature that provide the optimal bid for RES generation considering a hedging option based on a storage device; (3) an additional contribution of this paper is the sensitivity analysis performed on the results to evaluate the influence of BESS size and market prices on the relevance of using a PV/BESS. In particular, the optimal sizing of the BESS depending on the control strategy is discussed. We also determine the best strategy depending on market prices e.g. whether it is better to use a BESS only to compensate the forecast errors, or whether production should also be shifted to times with higher day-ahead prices.

The paper is structured as follows: in section 2, the assumptions we make for modeling the market are presented. Then sections 3 and 4 present the methods proposed for controlling the PV/BESS at the day-ahead and real-time stages, respectively. Section 5 describes the tools involved in these methods, and section 6 describes the test case. Section 7 shows the results, while the sensitivity analysis is performed in section 8. Finally, section 9 draws the conclusions of the paper.

\section{Market Structure}

In this paper, the market structure we consider comprises two parts:

- A day-ahead market where all actors can submit buying and selling orders up to $12 \mathrm{AM}$ before the day of delivery. The orders are then aggregated to make supply and demand curves and ultimately derive a day-ahead price $\pi$ for each market time unit.

- A balancing market where all actors must financially compensate any deviation between the amount of energy sold on the day-ahead market and the actual energy they have produced i.e. the imbalance. The compensation is derived after the day of delivery. The deviations are compensated with a balancing price which changes for each market time unit.

We consider a dual-price market. This means that the balancing prices are different depending on the sign of the imbalance. We note $\pi_{+}$the balancing price for positive imbalances (energy produced higher than energy sold), and $\pi_{-}$the balancing prices for negative imbalances (energy produced lower than energy sold). In such a situation, for a given time step, the revenue $R$ of a producer that sells an amount of energy $B$ (for Bid) but actually produces $E$ writes:

$$
R=\pi B+ \begin{cases}\pi_{+}(E-B) & \text { if } E>B \\ \pi_{-}(E-B) & \text { if } E<B\end{cases}
$$

It is useful to rewrite the revenue as:

$$
R=\pi E-\pi_{B}(E-B)
$$

with

$$
\pi_{B}= \begin{cases}\pi-\pi_{+} & \text {if } E>B \\ \pi-\pi_{-} & \text {if } E<B\end{cases}
$$

This formulation is useful because it clearly reflects the way the revenue is calculated. The first term of the equation is the revenue generated from selling the actual energy produced at the day-ahead price. The second term is a penalty term corresponding to the financial compensation of the imbalances. Usually, the balancing prices are defined such that $\pi_{+} \leq \pi \leq \pi_{-}$, so that this second term is positive.

Finally, to derive the control algorithms, we reformulate by differentiating the part of the production $E$ that comes from the PV panels $E_{P V}$ and the part that comes from the BESS $E_{B E S S}$.

We also introduce a term $C\left(E_{B E S S}\right)$, that reflects the costs due to aging of the BESS when used to deliver the amount of energy $E_{B E S S}$. This is obtained with the rainflow counting algorithm [17]. The aging of the BESS can be divided into two components, i.e. cycling aging and calendar aging, which is the degradation caused by time. In the remainder of the paper, we will focus on the cycling aging of the BESS and consider its calendar aging as a given lifetime. The end-of-life of the BESS is thus defined as the minimum lifetime given by the cycling and calendar aging. As an example, if the calendar aging gives a lifetime of 20 years, and the cycling aging a lifetime of 50 years, we consider that the actual lifetime of the BESS is 20 years (as opposed to considering that the cycling aging adds up to the 20 years given as the calendar lifetime).

We penalize the revenue with the cost associated with the lifeloss of the BESS. Note that the penalized revenue $R^{\prime}$ is not an actual cash flow, and that the cost associated with the life-loss is only here to make the control of the BESS more conservative regarding the lifetime. The penalized revenue $R^{\prime}$ then writes:

$$
\begin{aligned}
R^{\prime}=\pi\left(E_{P V}+E_{B E S S}\right) & -\pi_{B}\left(E_{P V}+E_{B E S S}-B\right) \\
& -C\left(E_{B E S S}\right)
\end{aligned}
$$

\section{Day-ahead Offering Strategy}

Different algorithms are required for day-ahead bidding of the PV/BESS, and for real-time control of the system. The aim of the first control algorithm is to provide the bids of the combined PV/BESS for the forthcoming day. We propose a first benchmark where the BESS is not used at the day-ahead level, and a second where the BESS is taken into account along with its usage cost.

In all the proposed algorithms, the PV/BESS is considered a price-taker. This means that we assume that the PV/BESS bids have no influence on the day-ahead price. This hypothesis seems reasonable since the generation of the PV/BESS is low compared to the typical volume of energy exchanged on electricity markets. However, the number of participant with uncertain production, usually having a price taker behavior, increases on electricity markets. Thus, the influence they have on the day-ahead prices becomes more and more significant. For the sake of simplicity, we do not model this influence in this paper.

\subsection{Benchmark: No BESS in the day-ahead planning}

For the benchmark, we do not use the BESS at the day-ahead level, and thus, all the terms related to the BESS are ignored. To derive the optimal bids $B_{D A}^{*}$, we must then solve:

$$
B_{D A}^{*}=\operatorname{argmax}_{B \in \mathbb{R}^{N}} \sum_{i=1}^{N} \pi_{i} E_{i}-\pi_{B, i}\left(E_{i}-B_{i}\right)
$$

where $N$ is the number of market time units in a day. In these conditions, it has been proven that the optimal bids that minimize the penalties for the producer are given by [1]:

$$
B_{D A, i}^{*}=F_{i}^{-1}\left(\frac{\pi_{i}-\pi_{+, i}}{\pi_{-, i}-\pi_{+, i}}\right)
$$

where $F_{i}$ is a forecast Cumulative Distribution Function (CDF) of the energy production of the plant for the $i$-th market time unit. The application of this strategy for a PV power plant thus requires a probabilistic PV power forecasting model, and a forecasting model of the day-ahead and regulation prices. This benchmark strategy is referred to as strategy DA0 in the remainder of the paper. 
When the BESS is used at both the day-ahead and real-time levels, then the entire formulation of the revenue from equation (4) is optimized. Once again, we separate the bids into one part accompanied by uncertainty from the PV plant $B_{P V}$, and the output from the battery $B_{B E S S}$. Since the BESS is controllable, we assume that the actual output of the BESS $E_{B E S S}$ is equal to the amount bid $B_{B E S S}$. With these assumptions, the optimization problem that needs to be solved to derive the optimal bids is:

$$
\begin{gathered}
B_{P V}^{*}, B_{B E S S}^{*}=\operatorname{argmax}_{\left[B_{P V} \in \mathbb{R}^{N}, B_{B E S S} \in \mathbb{R}^{N}\right]} \\
\sum_{i=1}^{N} \pi_{i}\left(E_{P V, i}+B_{B E S S, i}\right)-\pi_{B, i}\left(E_{P V, i}-B_{P V, i}\right) \\
-C\left(B_{B E S S, i}\right)
\end{gathered}
$$

However, to ensure that we can assume that $E_{B E S S}=B_{B E S S}$, and to correctly simulate the operation of a BESS, we must add several constraints. To define the constraints, we note as SOC (for State of Charge) the amount of energy in the battery at a given time step, relative to its full capacity $C a p$.

$$
\begin{gathered}
-\frac{1}{\eta_{C h}} \operatorname{Cap}\left(1-S O C_{i}\right)<B_{B E S S, i}<\eta_{\text {Dis }} \text { CapSOC }_{i} \\
-B_{B E S S}<\eta_{C h} E_{P V} \\
C a p\left|S O C_{i}-S O C_{i-1}\right| \leq K
\end{gathered}
$$

The first constraint ensures that the energy in the BESS is never lower than 0 or higher than the capacity of the battery, taking into account the charge and discharge rates of the BESS, respectively $\eta_{C h}$ and $\eta_{D i s}$. The second constraint ensures that the BESS can only be charged from the PV plant, and not from the grid. Finally, the third constraint is a limitation on the power rating of the BESS, defined by the parameter $\mathrm{K}$.

This strategy again requires probabilistic forecasts of the upcoming PV power production and forecasts of the day-ahead and balancing prices. In the remainder of the paper, this is referred to as strategy DA1.

\section{Real-time Control}

In real-time control of the PV/BESS, the algorithms are different. Since we are now in real time, the day-ahead prices and energy sold on the electricity market are known, and the only sources of uncertainty come from the PV power generation and balancing prices. Along with the benchmark strategy, which is to not use the BESS at all, we define two additional real-time control strategies.

The first one is purely analytical and tries to minimize the penalties for the next market time unit, without taking into account the BESS aging cost or the near future after the next market time unit. In contrast, the second strategy takes all of these factors into account.

\subsection{First strategy}

The first algorithm minimizes the term arising from imbalances between the bids and PV/BESS production. Since we are in real time, the bids $B_{D A}$ have already been submitted and the market has been cleared. Thus, the day-ahead prices $\pi$ are known and the only design variable is the BESS output $E_{B E S S}$. The BESS is allowed to deviate from its planning $B_{B E S S}$ to compensate deviations coming from the PV power forecast error, thus we do not necessarily have $E_{B E S S}=B_{B E S S}$ anymore. At this stage, the only design variable is the amount of energy we charge or discharge from the BESS $E_{B E S S}$. In this case, we can write the real-time revenue $R_{R T}$ as a function of $E_{B E S S}$ only and get:

$$
\begin{aligned}
R_{R T}\left(E_{B E S S}\right)= & E_{P V} \pi-\left(E_{P V}+E_{B E S S}-B_{D A}\right)\left(\pi-\pi_{B}\right) \\
& +E_{B E S S} \pi-C\left(E_{B E S S}\right)
\end{aligned}
$$

For the first method, we focus on reducing the penalties, so we neglect the term $\pi E_{B E S S}$ and the BESS usage costs $C\left(E_{B E S S}\right)$. The first neglected term represents a profit that can be obtained from the difference in day-ahead prices during the day. However, this profit is supposed to have already been realized at the day-ahead level. Besides, the profit alternates between positive and negative values depending on the charge or discharge of the BESS. Its impact should thus be reduced when summed over several time steps. On the other hand, the penalty term Pen= $\left(E_{P V}+E_{B E S S}-B_{D A}\right)\left(\pi-\pi_{B}\right)$ is always positive. Finally, neglecting the BESS usage costs allows us to propose a closed-form solution to the revenue maximization problem. The expectation of the penalty term Pen for the next time step writes:

$$
\mathbb{E}(\text { Pen })=\int_{0}^{E_{n}}\left(p+E_{B E S S}-B_{D A}\right)\left(\pi-\pi_{B}\right) f_{P V}(p) d p
$$

where $E_{n}$ is the maximum amount of energy that the plant can produce on a given time step, and $f_{P V}$ is the Probability Distribution Function (PDF) of the PV power. Since $\pi_{B}$ is dependent on the sign of the imbalance, the expectation of the penalty term must be rewritten:

$$
\begin{aligned}
\mathbb{E}(\text { Pen }) & =\int_{0}^{B_{D A}-E_{B E S S}}\left(p+E_{B E S S}-B_{D A}\right)\left(\pi-\pi_{-}\right) f_{P V}(p) d p \\
& +\int_{B_{D A}-E_{B E S S}}^{E_{n}}\left(p+E_{B E S S}-B_{D A}\right)\left(\pi-\pi_{+}\right) f_{P V}(p) d p
\end{aligned}
$$

Usually, the prices are defined so that imbalances that support the grid imbalance at the national level are not penalized. That is:

$$
\begin{aligned}
& \pi_{+}=\pi, \pi_{-}>\pi \text { if the grid is short of energy } \\
& \pi_{+}<\pi, \pi_{-}=\pi \text { if the grid contains excess energy }
\end{aligned}
$$

We assume that we have an estimation of the probability $p_{G}$ for the grid to fall short on the national level. We can then substitute the forecast regulation prices $\pi_{+}, \pi_{-}$with random variables $\pi_{p o s}, \pi_{n e g}$ modeled by a sum of Dirac distributions:

$$
\begin{aligned}
\pi_{p o s}(x) & =p_{G} \delta(x-\pi)+\left(1-p_{G}\right) \delta\left(x-\pi_{+}\right) \\
\pi_{n e g}(x) & =p_{G} \delta\left(x-\pi_{-}\right)+\left(1-p_{G}\right) \delta(x-\pi)
\end{aligned}
$$

which gives:

$$
\begin{gathered}
\mathbb{E}(\text { Pen })=p_{G}\left(\pi-\pi_{-}\right) \int_{0}^{B_{D A}-E_{B E S S}}\left(p+E_{B E S S}-B_{D A}\right) f_{P V}(p) d p \\
+\left(1-p_{G}\right)\left(\pi-\pi_{+}\right) \int_{B_{D A}-E_{B E S S}}^{E_{n}}\left(p+E_{B E S S}-B_{D A}\right) f_{P V}(p) d p
\end{gathered}
$$

Using the variable change $x=p-B_{D A}$, we get: 


$$
\begin{aligned}
& \mathbb{E}(\text { Pen })=p_{G}\left(\pi-\pi_{-}\right) \int_{0}^{-E_{B E S S}}\left(x+E_{B E S S}\right) f_{P V}\left(x+B_{D A}\right) d x \\
& \quad+\left(1-p_{G}\right)\left(\pi-\pi_{+}\right) \int_{-E_{B E S S}}^{E_{n}-B_{D A}}\left(x+E_{B E S S}\right) f_{P V}\left(x+B_{D A}\right) d x
\end{aligned}
$$

Finally, using the Leibniz rule for differentiating under the integral sign, we obtain:

$$
\begin{aligned}
\frac{d \mathbb{E}(\text { Pen })}{d E_{B E S S}}= & p_{G}\left(\pi-\pi_{-}\right) F_{P V}\left(B_{D A}-E_{B E S S}\right) \\
& +\left(1-p_{G}\right)\left(\pi-\pi_{+}\right)\left(1-F_{P V}\left(B_{D A}-E_{B E S S}\right)\right.
\end{aligned}
$$

The second derivative is:

$$
\begin{aligned}
\frac{d^{2} \mathbb{E}(P e n)}{d E_{B E S S}^{2}}= & -p_{G}\left(\pi-\pi_{-}\right) f_{P V}\left(B_{D A}-E_{B E S S}\right) \\
& +\left(1-p_{G}\right)\left(\pi-\pi_{+}\right) f_{P V}\left(B_{D A}-E_{B E S S}\right)
\end{aligned}
$$

This second derivative is always positive by definition of the regulation prices. Thus, by making the first derivative equal to 0 , we find the minimum:

$$
E_{B E S S}^{*}=B_{D A}-F_{P V}^{-1}\left(\frac{1}{1+\frac{p_{G}}{1-p_{G}} \frac{\pi_{-}-\pi}{\pi-\pi_{+}}}\right)
$$

The first method is then to compute a forecast distribution of the PV power, deterministic forecasts of the regulation prices, and the probability that the system will fall short, and to inject them into this optimal solution. Although the solution is in a closed form, the BESS constraints prevent the use of this solution more than one time step ahead, and the BESS usage cost is neglected. This is referred to hereafter as the RT1 method.

\subsection{Second strategy}

The second method is very similar to the offering strategy including the BESS from section 3.2, however it is performed using a Model Predictive Control (MPC) approach, to adapt it to the real time. This means that the whole revenue formulation is maximized over the $N_{M P C}$ next time steps, then the result of the optimization from the first time step is used as the command for the BESS for the next market time unit. This allows us to take into account the future forecast state of the system in the real-time control.

Since we are in a real-time setting, the day-ahead prices and bids are known, as for the first real-time strategy. As a result, the only design variable is the BESS command. Therefore, the optimization problem to solve for each time step is:

$$
\begin{aligned}
& E_{B E S S}^{*}=\operatorname{argmax}_{E_{B E S S}} \in \mathbb{R}^{N_{M P C}} \\
& \begin{aligned}
\sum_{i=1}^{N_{M P C}}\left(E_{P V}+E_{B E S S}\right) \pi & -\left(E_{P V}+E_{B E S S}-B_{D A}\right)\left(\pi-\pi_{B}\right) \\
& -C\left(E_{B E S S}\right)
\end{aligned}
\end{aligned}
$$

subject to the same constraints as in section 3.2. We also add the constraint that the day-ahead BESS schedule must remain feasible over the $N_{M P C}$ next time steps after the operation of the BESS, which translates by:

$$
-\frac{1}{\eta_{C h}} \operatorname{Cap}\left(1-S O C_{i}\right) \leq B_{B E S S, i} \leq \eta_{\text {Dis }} \text { CapSOC }_{i}
$$

We change $N_{M P C}$ at each time step, depending on the time of day, so that all of the remaining day is included in the optimization. This is especially important because day-ahead planning often results in full discharge of the BESS in the evening when day-ahead prices are usually high due to high demand. As such, the whole day must be included in the optimization loop. If $N_{M P C}$ is too low, the BESS could discharge itself entirely during the day to compensate forecast errors, and thus be unable to provide the energy in the evening. This second method is referred to as RT2.

\section{Forecasting and Optimization Tools}

To implement these different algorithms, several supplementary models are required. They are presented in this section.

\subsection{Probabilistic forecasts for PV production}

One of the most important models required is the PV power forecast model. All approaches require probabilistic forecasts of PV power production.

The model we implemented is based on the Analog Ensemble (AnEn) proposed in [18]. We extended it in several ways to improve its performance and allow it to produce reliable forecasts for both short-term and long-term forecasts, and with different time resolutions.

The main extensions are:

- The inclusion of different data sources such as measurements and satellite data, in addition to Numerical Weather Predictions (NWPs) that are the only data source in the original model.

- A dynamic algorithm to compute the relevance of each source of data depending on the forecast start time and horizon, so that the weight of the different sources varies over time. For example, the forecasts rely much more on measurements for forecasts with horizons under one hour, or on NWPs for day-ahead forecasts.

This model is well suited to control algorithms, since it can provide forecasts with both high resolutions (up to 1 minute) for real-time control strategies, and long horizons (up to 48 hours) for day-ahead planning algorithms. [19].

The PV power forecasting model is presented in more detail in

\subsection{Market quantity forecasts}

Day-ahead planning algorithms require forecasts of both day-ahead and balancing prices. Real-time control algorithms also require forecasts of balancing prices.

day-ahead prices are obtained with a Support Vector Regression (SVR) model, which is commonly used for energy price forecasting [20]. The inputs of the regression are the forecasts of national demand and renewable energy generation for the next day, along with the month and day of the week.

In the next step, the balancing price forecasts are obtained by applying $\mathrm{k}$ nearest neighbor (k-NN) methodology to the day-ahead price. In real time, since the day-ahead prices are known, the k-NN model is applied again using the actual day-ahead prices to update the balancing price forecasts.

\subsection{Optimizer}

To solve the different optimization problems that appear in the control algorithms, we decide to employ stochastic optimization, since we already dispose of probabilistic forecasts of the PV power generation. A large number of PV production scenarios are generated 
following [21]. The scenarios are then reduced using a Partitioning Around Medoids (PAM) algorithm, and the median of the objective over the scenarios is optimized. The resulting non-linear optimization problem is solved using the COBYLA algorithm [22].

The PAM algorithm reduce the scenarios by partitioning the whole set of scenarios in a fixed number of classes $k$. The algorithm can be summarised as follows:

- Compute the distance between each pairs of scenarios. In the paper, we used the sum of the euclidean distances between the realizations as the distance. In other words, given two scenarios $P_{1, \ldots t}$ and $P_{1, \ldots, t}^{\prime}$, the distance between the scenarios is given by $D=\sum_{i=1}^{t} \sqrt{\left(P_{i}-P_{i}^{\prime}\right)^{2}}$

- Find $k$ scenarios that are representative of the whole scenarios, called medoids. To do so, the sum of the distances between each scenario and its closest medoid is minimized using an heuristic optimization algorithm.

- Associate each scenario to its closest medoid.

Then, the medoids are used as probable scenarios, and the probability of each medoid is estimated by the number of scenarios populating this medoid's class compared to the total number of scenarios.

We considered it important to use scenarios to represent the uncertainty because of the temporal dimension of the PV/BESS management problem. This temporal dimension can be seen from the second constraint formulated in equation (9). One of the essential characteristics of PV power forecasts is the positive correlation between the forecast errors at consecutive time steps. In other words, if a forecast error is positive (resp. negative) for a given time step, the forecast error for the following time step is also likely to be positive (resp. negative). This is a problem for BESSs, because since a BESS can compensate forecast errors, a significant error present on several consecutive time steps would quickly either charge the BESS to its maximum or discharge it to its minimum, depending on the sign of the error. Due to the temporal correlation of the errors, this worstcase scenario is much more likely than the consecutive distributions might suggest if they were considered independent. During the simulation, the energy remaining in the BESS is tracked to ensure that the second constraint from equation (9) is respected.

\section{Test Case}

A simulation of the control of the PV/BESS is performed for four months (January to May 2017)for a large PV plant located in France. The plant is situated at longitude -0.9223889 , latitude 44.19025 with an elevation of 72 meters above sea level, and has an installed power of $9828 \mathrm{kWp}$.

The entire control is carried out taking an MPC approach. For each time step, the PV power and market quantity forecasts are updated based on the inputs known at the time. Then, if the dayahead market closes for the considered time step, bids are submitted for the next day using one of the two methods from section 3 . The control set-point for the next time step is obtained using one of the two methods from section 4 . Then, the process goes to the next time step, updates the BESS State Of Charge (SOC), the PV power, and market quantities forecast, and continues the algorithm until the final time step. A flowchart of the algorithm is represented on Fig. 1.

The NWPs required for the AnEn model are obtained from the European Center for Medium-range Weather Forecasting (ECMWF), along with measurements and satellite data to improve short-term forecasts. Forecasts of the national demand and renewable energy generation required for the day-ahead price forecasts are provided by RTE, the French Transmission System Operator (TSO).

The BESS considered in the test case is a lithium-ion storage system. Aging parameters for the rainflow counting algorithm are taken from [23] and [24]. Regarding costs, prospective values for the year 2030 from [24] are used in the base case, that is a $200 € / \mathrm{kWh}$ investment cost. Besides, in all simulations, we set the parameter $K$, which controls the power rating of the BESS so that the BESS can fully

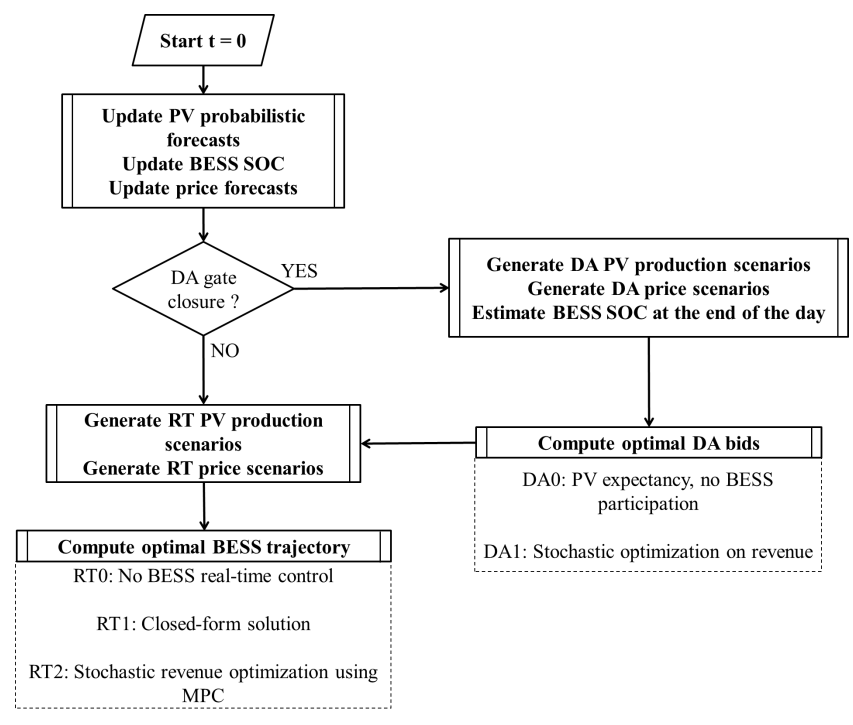

Fig. 1: Flowchart of the overall optimization approach

charge or discharge in two hours. This is to simulate a BESS with a power rating of $0.5 \mathrm{C}$, which is common in commercial lithium-ion storage systems.

The simulation is performed on the software R, using the packages e1071 [25] for the SVR model and nloptr [26] for the implementation of the COBYLA algorithm.

Different combinations of day-ahead and real-time methods are evaluated. The sensitivity of the results to the installed capacity of the BESS and its investment costs is studied, providing guidelines on the sizing of the BESS for such applications. The different method combinations tested are summarized in table 1.

An example of the typical output from the four strategies is represented on Fig. 2. We can see that strategies S1 and S2 focus only on reducing the imbalance. Strategy S3 tends to reduce the imbalance but also shifts the production to benefit from high prices in the evening. Finally, strategy S4 spends most of the day charging the BESS and then entirely discharges it in the evening.

\section{Results}

\subsection{Performance of the forecasting models}

The PV power model's performance over the testing period is represented on Fig. 3. The graphs show the Mean Absolute Error (MAE) and the bias of the model for different forecast horizons normalized using the PV plant's installed capacity $P n$. For the sake of simplicity, only a deterministic analysis of the results instead of a thorough probabilistic evaluation is performed, taking the expectancy of the forecasted distributions as a deterministic forecast. These indicators are defined as follows for a set of $N_{f}$ forecasts and targets $\{\hat{y}, y\}$ :

Table 1 Evaluated strategies

\begin{tabular}{lll}
\hline Strategy & DA bidding & RT control \\
\hline S0 (benchmark) & DA0 & RT0 \\
S1 & DA0 & RT1 \\
S2 & DA0 & RT2 \\
S3 & DA1 & RT1 \\
S4 & DA1 & RT2 \\
\hline
\end{tabular}




$$
\begin{aligned}
& M A E=\frac{1}{N_{f}} \sum_{i=1}^{N_{f}}|y-\hat{y}| \\
& B I A S=\frac{1}{N_{f}} \sum_{i=1}^{N_{f}}(y-\hat{y})
\end{aligned}
$$

To understand further the behavior of the control models, the MAE and biases of the price forecasting models are reported on table 2.

Table 2 Performance of the price forecasting models

\begin{tabular}{lc|cc|cc}
\hline & day-ahead & \multicolumn{2}{|c|}{ Balancing - positive errors } & \multicolumn{2}{c}{ Balancing - negative errors } \\
\hline & & Day-ahead & Intra-day & Day-ahead & Intra-day \\
BIAS (\%) & 16.5 & 17.8 & 0.77 & 17.9 & 1.32 \\
MAE (\%) & 20.1 & 26.6 & 16.4 & 27.3 & 22.2 \\
\hline
\end{tabular}

The results show that the shorter the forecast horizon, the lower the error. This intuitive result confirms our intuition in differentiating between a day-ahead and a real-time control algorithm, since the forecast updates improve the forecast accuracy in the short-term.

The price forecasts are subject to greater forecast errors. However, the quantity of interest for the control algorithm is the relative magnitude of the balancing prices compared to the day-ahead prices, and not the absolute forecast value. Since the balancing price forecasts are subject to a similar bias between positive and negative errors, the relative magnitudes should be preserved. Still, the values reported are only averages and not fully representative of the model's behavior, and price forecast errors can still lead to counterproductive behavior e.g. by compensating an imbalance that would not have

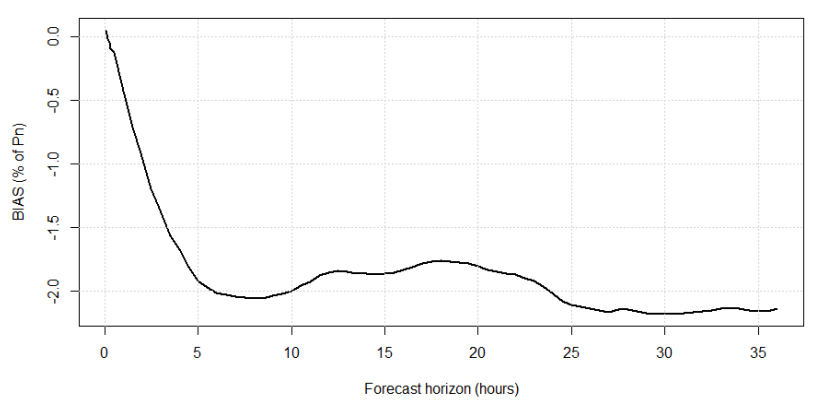

(a) BIAS

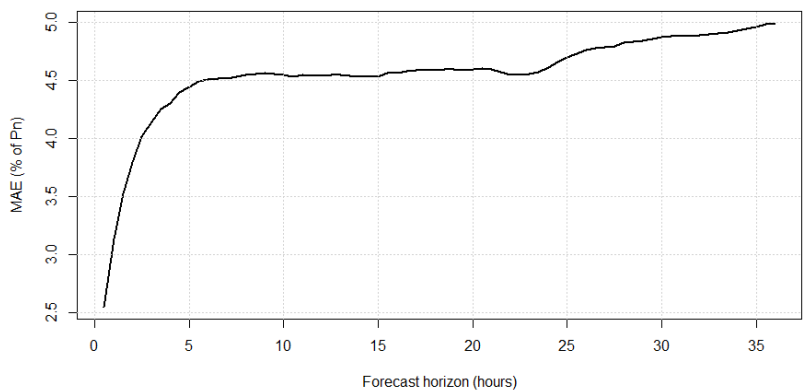

(b) MAE

Fig. 3: Performance of the PV power forecasting model

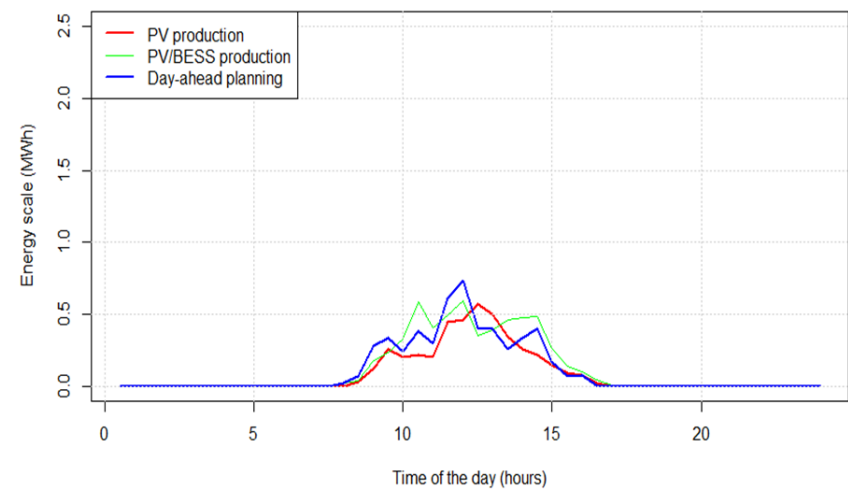

(a) Strategy S1

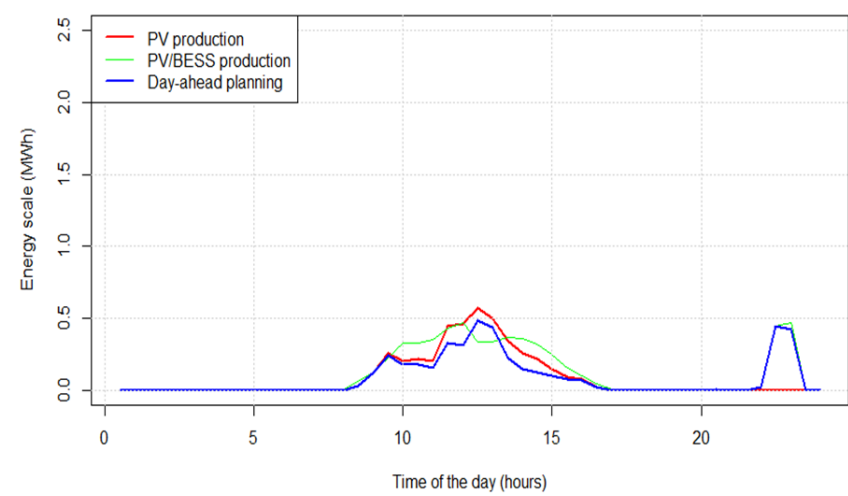

(c) Strategy S3

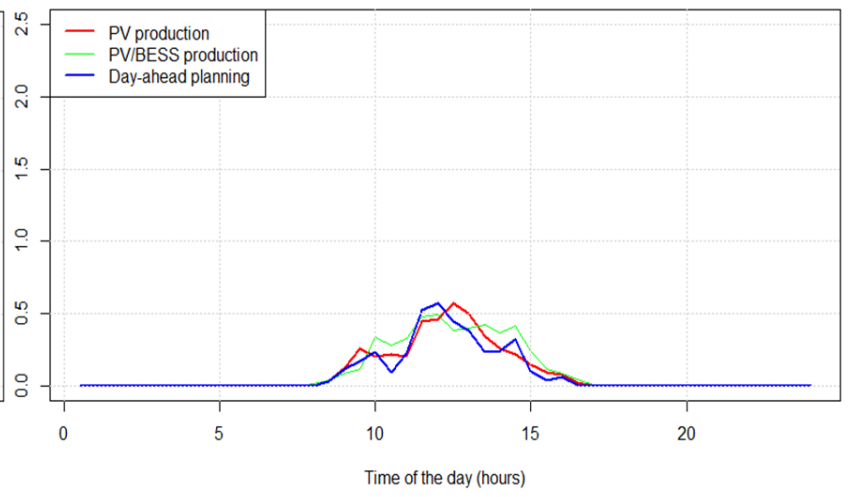

(b) Strategy S2

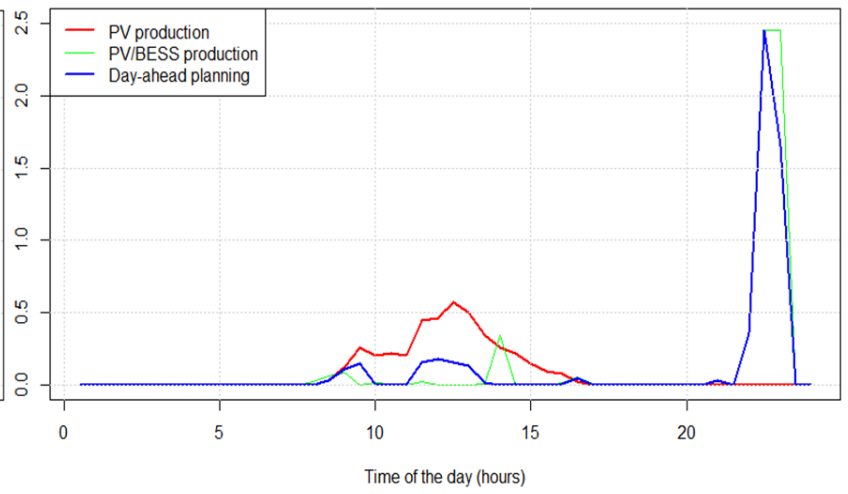

(d) Strategy S4

Fig. 2: Example outputs from the four strategies, for day 2017-01-16 
been penalized, or ignoring an imbalance that is actually penalized much more than expected. Using a representation of the uncertainty of the price forecasts would definitely improve this aspect, by lowering the "trust" that the control algorithms would put in a given value as a deterministic forecast of the prices.

The bias of the models also offers relevant information to explain the control algorithms. As shown on Fig. 3, the model develops a slight bias when the forecast horizon increases, and thus tends to overestimate the production. Thus, the bids have a tendency to be higher than the actual production. Ultimately, this results in the BESS having to discharge more frequently than charge, which facilitates the emergence of saturation effects.

\subsection{Test Case Results}

The results of the study are shown on table 3 . The results presented feature actual cash flows i.e. the revenue indicated in the table does not include the cost associated with the BESS life loss. The purpose of this cost is only to help the algorithms controlling the BESS in a more conservative way.

Table 3 Evaluation Results

\begin{tabular}{ccccc}
\hline & $\mathrm{S} 1$ & $\mathrm{~S} 2$ & $\mathrm{~S} 3$ & $\mathrm{~S} 4$ \\
\hline Error reduction (\%) & 9.6 & 11.6 & -0.47 & -5.5 \\
Penalty reduction (\%) & 13.5 & 15.5 & 12.9 & 9.11 \\
Revenue increase (\%) & 1.09 & 0.53 & 3.52 & 2.60 \\
BESS life loss (\%) & 0.08 & 0.11 & 0.43 & 0.38 \\
\hline
\end{tabular}

We can see from the results that only strategies S1 and S2 contribute to reducing the imbalance. With strategies S3 and S4, the total imbalance is actually increased compared to the case without BESS. Since the BESS is used in day-ahead trading, its available capacity to compensate imbalances is decreased. Besides, if the day-ahead forecast errors are high, then the BESS cannot fulfill its day-ahead commitment. For example, if the forecasts overestimate the PV power generation and the BESS is scheduled to discharge entirely in the evening, the lower amount of PV production prevents the BESS from realizing its commitment because it cannot charge up to the required SOC before discharging. This ultimately results in a higher imbalance. Since the PV power forecasting model has a tendency to overestimate production, this is a common scenario.

Reducing the imbalance more naturally results in a greater imbalance reduction for strategies S1 and S2. It is remarkable that strategy $\mathrm{S} 2$ is better at reducing both the error and revenue compared to $\mathrm{S} 1$. This is the direct influence of taking into account the near future when managing the BESS, as the control considers both the upcoming residual imbalance and price forecasts.

However, despite the increased imbalance, strategies S3 and S4 feature a much higher revenue increase. This is because they profit from the differences in day-ahead price at different times of the day, which is impossible with strategies S1 and S2 where the BESS does not participate in the day-ahead bidding. The revenue stream from this source of profit is much more reliable than from the compensation of imbalances, because the day-ahead price behavior is much less volatile, and it has a marked daily pattern featuring higher prices in the morning and evening when energy demand is high. As a result, the revenue from strategies S3 and S4 is much higher despite their increased imbalance. Overall, the strategy that leads to the highest increase in revenue is strategy $\mathrm{S} 3$.

Finally, the BESS degradation is quite low for all strategies, although greater for strategies S3 and S4 where deep charging and discharging cycles are performed. Overall, BESS life loss is very low. In the worst case, the BESS life loss is $0.43 \%$ over the 4 months of the testing period, which means that the BESS would last around 75 years considering only the cycling life loss. In practice, calendar aging will reduce the lifetime of the BESS and cause its end of life much earlier, typically after 10-20 years of usage [27].

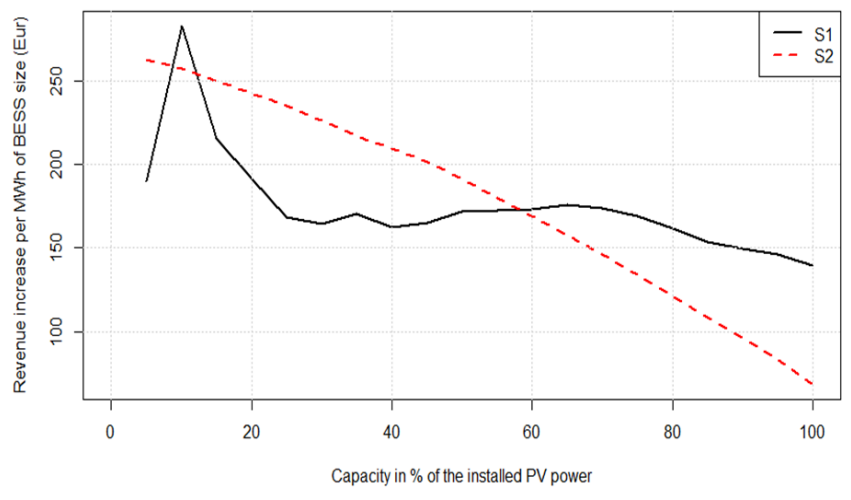

(a) Strategies S1 and S2

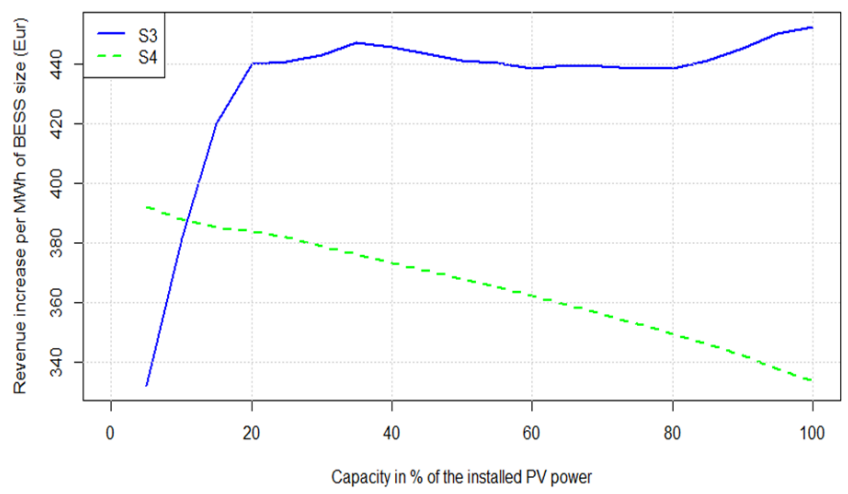

(b) Strategies $\mathrm{S} 3$ and $\mathrm{S} 4$

Fig. 4: Sensitivity to BESS size

\section{Sensitivity Analysis}

In this section, we discuss the sensitivity of the results to different parameters of the simulation. To perform the sensitivity analysis, we extrapolated the raw results from the initial simulations. We used different methods depending on the strategy and the parameter for which we evaluate the sensibility.

\subsection{Sensitivity to the BESS dimension}

This analysis was based on our observation that BESS life loss from cycling aging is very low compared to the calendar aging. Therefore, we neglected the BESS aging in the objective function from equation (4). Following this, we noticed that both the objective function and the constraints were linear with respect to the BESS capacity. Thus, for the optimization problems for DA1 and RT2, we extrapolated that reducing the BESS bids $B_{B E S S}$ by the same factor as the BESS size reduction would provide a good estimate of the optimal bids, while still respecting the constraints. Note however that this approach is only valid for BESS sizes lower than the size of the initial simulation, because the constraint from equation (9) remains true for lower bids. However, if the BESS size and thus the BESS optimal bids increase, this constraint could be strongly violated.

For the algorithms DA0 and RT1, the decisions are independent from the BESS size. Thus we only have to calculate the simulation results with the same commands as the control algorithm, but ensuring that the constraints are respected to show the saturation effects from the BESS.

The sensitivity of the results to the BESS size is reported on Fig. 4. As revenue, imbalance and penalties increase almost linearly with the installed capacity, we represent the revenue increase per capacity installed to better reflect the efficiency of the different approaches.

For strategies S1 and S2, it seems that strategy S2 is more efficient for lower BESS sizes, while S1 is better for bigger BESSs. This is understandable, as strategy $\mathrm{S} 2$ takes into account the current SOC of the BESS when controlling it, and thus is better at managing 
small BESSs where BESS saturation occurs much more often. On the contrary, in larger BESSs, these saturation effects are much less frequent, and strategy S1 performs better, as it has behaves more greedily i.e. it ignores the BESS life loss and maximizes instantaneous profit, without saturation effects. Using PV power forecasting models with a lower bias, the size for which the saturation effects become significant could be reduced, allowing easier management of small BESSs.

There is, however, an exception for a BESS size of around $10 \%$ of the installed PV power. It seems that for this ratio, the most critical imbalances are compensated, and any increase in BESS size generates marginally less profit. With strategy S2, more parameters than only the price signals are included in the control, which is actually detrimental to this BESS ratio. Overall, the revenue increase per BESS size is smoother with strategy S2, and we expect that this peak for strategy $\mathrm{S} 1$ is largely dependent on the test data. In any case, the larger the BESS, the lower the amount of revenue generated per installed BESS capacity. This suggests that the best strategy when the BESS is not allowed to participate in the day-ahead market is to install a small BESS, controlled with strategy S2.

For strategies S3 and S4, the same effects appear: strategy S4 performs better for small BESSs, then is outperformed by the more greedy S3 strategy for larger BESSs when the saturation effects are less frequent. However, unlike with strategies S1 and S2, a larger BESS generates more revenue for strategy S3. It thus seems preferable to install larger BESSs, controlled by strategy S3 when they can take part in the day-ahead market.

Overall, it appears from the results that taking BESS life loss into account does not generate a significant improvement in control strategies. Besides, taking the near future into account when managing a BESS is only useful when the ratio of BESS capacity compared to PV capacity is low (that is, $\leq 10 \%$ ). For higher ratios, the saturation effects are less frequent and thus a simpler algorithm can be used.

Besides, it seems that the market conditions of the test case are not favorable to PV/BESS. Compared to the $200,000 € / M W h$ we used as the installation price of a BESS, gaining at most 450 €over the course of the test period ( 4 months) leads to an extremely long return on investment.

\subsection{Sensitivity to market conditions}

Finally, we established that one parameter was of particular importance for analyzing the results, and that was the market conditions. Namely, we compared the difference in results when the day-ahead prices are higher, and when the magnitude between the day-ahead prices and balancing prices is higher.

However, we assumed that the controlling algorithms' output was the same after modifying the price signals. This assumption is true only for strategies S1 and S3, if we keep the same relative magnitude between the day-ahead and balancing prices, so that the perception of the financial risk of being in imbalance is the same for the different algorithms. For strategies S2 and S4, since other quantities than the prices are involved in the real-time controlling algorithms, it is not possible to modify the price signals without modifying the control of the BESS.

We tested the strategies on two variations of the price signals. The results are represented on Fig. 5. The first variation we tested involved multiplying the day-ahead prices by a given factor, maintaining the same difference between the day-ahead and balancing prices ("day-ahead only"). The second variation was to multiply the difference between the balancing prices and the day-ahead prices by a given factor, keeping the same day-ahead signal ("Balancing only"). Finally, we can also multiplied both signals ("day-ahead and balancing"). However, we observed that the multiplication factor must remain the same so that the relative difference between day-ahead and balancing prices remains the same.

Naturally, strategy S1 only benefits when the balancing prices are modified, since it only focuses on compensating the penalties. Moreover, it benefits even greater when the gap between the day-ahead and balancing increases without increasing the day-ahead prices, as

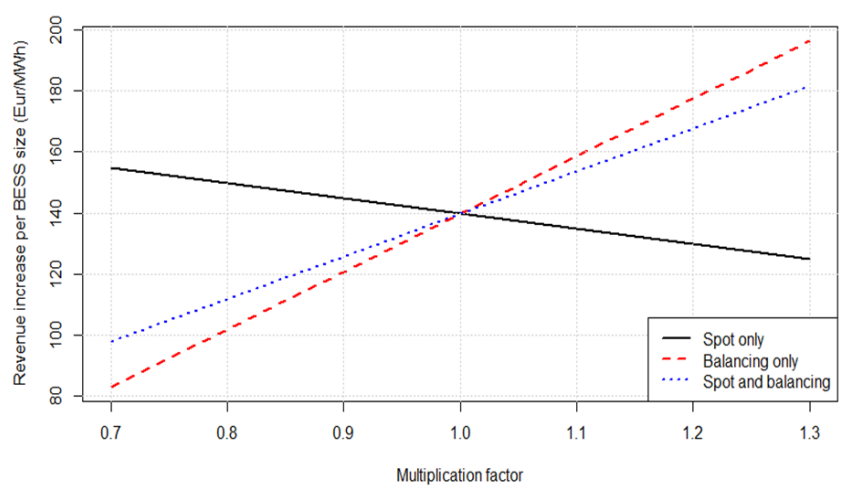

(a) Strategy S1

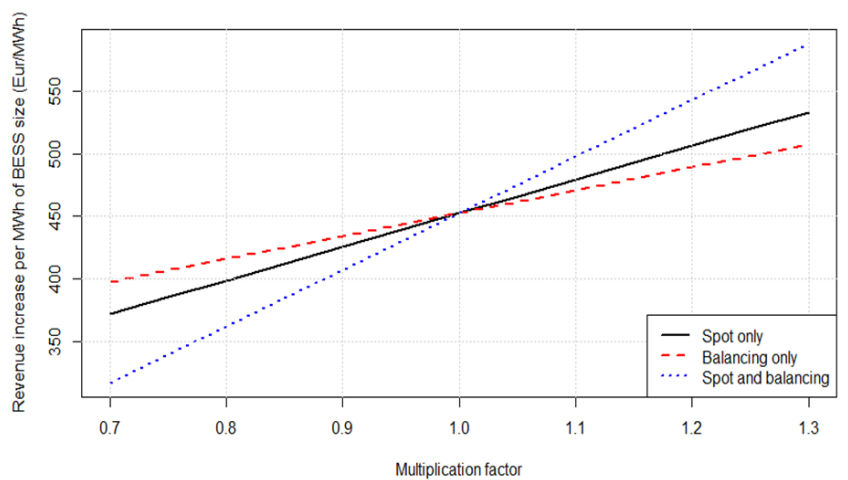

(b) Strategy S3

Fig. 5: Sensitivity of the results to market conditions

the relative size of the penalties compared to the day-ahead revenue is lower when the day-ahead price increases. For strategy S1 to be optimal, it is thus better to have a market with significant balancing prices compared to the day-ahead prices i.e. that penalizes the imbalances more.

Strategy S2 benefits from increasing all price signals, since it deals with both day-ahead revenue and imbalance compensation. However, the marginal increase of revenue when increasing only the day-ahead prices is higher than when increasing only the balancing prices. Thus, it seems that a larger share of the revenue increase comes from the day-ahead revenue rather than from the imbalance compensation. Overall, strategy S3 is efficient with high electricity prices, and high imbalance compensation.

For both strategies, the revenue increase is at best directly proportional to the market prices. Since it is also directly proportional to the BESS installation cost, the return on investment time of a PV/BESS could be significantly reduced if both the BESS installation cost and the prices increase. Depending on whether the day-ahead or balancing prices increase, or both, this would result in different optimal control strategies.

\section{Conclusion}

In this paper, several strategies have been studied for the participation of a PV power plant coupled with a BESS in a dual-price electricity market. The different strategies include stochastic methods to consider upcoming PV power generation uncertainty, day-ahead and balancing price forecasts, and BESS life loss in the control of PV/BESS. We also studied whether it is preferable to use BESS capacity to shift production in day-ahead bidding, or to focus only on reducing imbalances caused by the forecast errors of PV power forecasting models.

The different strategies were evaluated on a 4-month test case. Analysis of the raw results, along with a sensitivity analysis on both the BESS size and the market conditions highlighted several 
aspects of the management of a PV/BESS. The main findings are the following:

- Due to the frequent small cycling of the battery, cycling aging is low compared to the calendar aging of a lithium-ion battery. Thus, this component can be neglected and it seems more profitable to maximize the usage of the BESS without taking into account its life loss.

- A BESS can efficiently contribute to the reduction of a plant's imbalance and penalties. However, since the gap between balancing prices and day-ahead prices is quite low and more unreliable compared to the gap between the day-ahead prices at different times of a day, it is much more efficient in terms of revenue to use the BESS for shifting the production at the day-ahead level, instead of reducing the imbalance.

- In the context of participation in day-ahead and balancing markets, the saturation effects of a BESS seem to become significant for BESS capacities below $1 \mathrm{MWh}$ for $10 \mathrm{MW}$ of PV power. Thus, the current SOC of BESSs must be taken into account in the controlling algorithm for these capacities. When the installed BESS is higher, the saturation effects still appear but they are much less detrimental to the benefits, so that is easier to ignore the current SOC in the control algorithm.

- Overall, the benefit of installing a BESS is very low compared to the installation cost of a BESS. The sensitivity analysis reveals that this benefit could be significantly improved by both a lower BESS cost, and higher day-ahead and balancing prices. Still, other revenue streams for the BESS should definitely be studied, namely participating in ancillary services markets.

\section{Acknowledgment}

The authors would like to thank the European Center for MediumRange Weather Forecasting (ECMWF) for providing the weather forecasts used in this study for the PV power forecasting model.

\section{References}

1 Pinson, P., Chevallier, C., Kariniotakis, G.N. 'Trading Wind Generation from Short-term Probabilistic Forecasts of Wind Power', IEEE Transactions on Power Systems, (2007), 22, (3), pp.1148-1156

2 Bourry, F., Juban, J., Costa, L.M., Kariniotakis, G. 'Advanced Strategies for Wind Power Trading in Short-term Electricity Markets'. In: European Wind Energy Conference. (Brussels, 2008).

3 Bitar, E.Y., Rajagopal, R., Khargonekar, P.P., Poolla, K., Varaiya, P.: 'Bringing wind energy to market', IEEE Transactions on Power Systems, (2012), 27, (3), pp.1225-1235

4 He, G., Chen, Q., Kang, C., Member, S., Pinson, P., Member, S., et al.: 'Optimal Bidding Strategy of Battery Storage in Power Markets Considering PerformanceBased Regulation and Battery Cycle Life', IEEE Transactions on Smart Grid, (2016), 7, (5), pp.2359-2367

5 Vespermann, N., Delikaraoglou, S., Pinson, P. 'Offering Strategy of a Price-Maker Energy Storage System in Day-Ahead and Balancing Markets'. In: PowerTech 2017. (Manchester, 2017).

6 Castronuovo, E.D., Usaola, J., Bessa, R., Matos, M., Costa, I., Bremermann, L., et al.: 'An integrated approach for optimal coordination of wind power and hydro pumping storage', Wind Energy, (2014), 17, (6), pp.829-852

7 Bourry, F., Costa, M., Kariniotakis, G. 'Risk-Based Strategies for Wind / PumpedHydro Coordination under Electricity Markets'. In: PowerTech. (Bucharest, Romania, 2009)

8 Perez, E., Beltran, H., Aparicio, N., Rodriguez, P.: 'Predictive Power Control for PV Plants With Energy Storage', IEEE Transactions on Sustainable Energy, (2013), 4, (2), pp.482-490

9 Abdeltawab, H.H., Mohamed, Y.A.R.I.: 'Market-Oriented Energy Management of a Hybrid Wind-Battery Energy Storage System Via Model Predictive Control With Constraint Optimizer', IEEE Transactions on Industrial Electronics, (2015), 62 (11), pp.6658-6670

10 Damiano, A., Gatto, G., Marongiu, I., Porru, M., Serpi, A.: 'Real-Time Control Strategy of Energy Storage Systems for Renewable Energy Sources Exploitation', IEEE Transactions on Sustainable Energy, (2014), 5, (2), pp.567-576

11 Saez-de-Ibarra, A. and Herrera, V. I. and Milo, A. and Gaztañaga, H. and Etxeberria-Otadui, I. and Bacha, S. and Padrós, A. : 'Management Strategy for Market Participation of Photovoltaic Power Plants Including Storage Systems', IEEE Transactions on Industry Applications, (2016), 52, (5), pp.4292-4303

12 Núñez-Reyes, Amparo and Marcos Rodríguez, David and Bordons Alba, Carlos and Ridao Carlini, Miguel Ángel: 'Optimal Scheduling of Grid-connected PV Plants with Energy Storage for Integration in the Electricity Market', Solar Energy, (2017), 144, pp.502-516

13 Conte, F., Massucco, S., Silvestro, F.: 'Day-ahead planning and real-time control of integrated pv-storage systems by stochastic optimization', IFAC-PapersOnLine, (2017), 50, (1), pp.7717-7723

14 Conte, F., Massucco, S., Saviozzi, M., Silvestro, F. 'A Stochastic Optimization Method for Planning and Real-Time Control of Integrated PV-Storage Systems : Design and Experimental Validation', IEEE Transactions on Sustainable Energy, (2018), 9, (3), pp.1188-1197

15 Keerthisinghe, C., Verbič, G., Chapman, A.C. 'Evaluation of a multi-stage stochastic optimisation framework for energy management of residential pv-storage systems'. In: Australasian Universities Power Engineering Conference (AUPEC). (Perth, Australia, 2014). pp. 1-6

16 Ding, H., Pinson, P., Hu, Z., Song, Y.: 'Optimal Offering and Operating Strategies for Wind-Storage Systems with Linear Decision Rules', IEEE Transactions on Power Systems, (2017), 31, (6), pp.4755-4764

17 Musallam, M., Johnson, C.M.: 'An Efficient Implementation of the Rainflow Counting Algorithm for Life Consumption Estimation', IEEE Transactions on Reliability, (2012), 61, (4), pp.978-986

18 Alessandrini, S., Delle Monache, L., Sperati, S., Cervone, G.: 'An Analog Ensemble for Short-term Probabilistic Solar Power Forecast', Applied Energy, (2015), 157, pp. $95-110$

19 Carriere, T., Vernay, C., Pitaval, S., Kariniotakis, G.: 'A novel approach for probabilistic photovoltaic power forecasting covering multiple time frames', IEEE Transactions on Smart Grid, (2019),

20 Weron, R.: 'Electricity Price Forecasting: A Review of the State-of-the-art With a Look Into the Future', International Journal of Forecasting, (2014), 30, (4), pp.1030-1081

21 Golestaneh, F., Gooi, H., Pinson, P.: 'Generation and Evaluation of Space-time Trajectories of Photovoltaic Power', Applied Energy, (2016), 176, pp.80-91

22 Powell, M.J. 'A Direct Search Optimization Method that Models the Objective and Constraint Functions by Linear Interpolation'. In: Advances in Optimization and Numerical Analysis. (Springer, 1994). pp. 51-67

23 Duggal, I., Venkatesh, B.: 'Short-term scheduling of thermal generators and battery storage with depth of discharge-based cost model', IEEE Transactions on Power Systems, (2015), 30, (4), pp.2110-2118

24 Fuchs, G., Lunz, B., Leuthold, M., Sauer, D.U. 'Technology Overview on Electricity Storage: Overview on the Potential and on the Deployment Perspectives of Electricity Storage Technologies'. (ISEA - Institut für Stromrichtertechnik und Elektrische Antriebe, 2012).

25 Meyer, D., Dimitriadou, E., Hornik, K., Weingessel, A., Leisch, F.. 'e1071: Misc Functions of the Department of Statistics, Probability Theory Group (Formerly: E1071)'. (TU Wien, 2017). R Package Version 1.6-8

26 Johnson, S.G.. 'The NLopt Nonlinear-optimization Package'. (MIT, 2018). http://ab-initio.mit.edu/nlopt

27 Rydh, C.J., Sandén, B.A.: 'Energy Analysis of Batteries in Photovoltaic Systems. Part I: Performance and Energy Requirements', Energy Conversion and Management, (2005), 46, (11-12), pp.1957-1979 